Pacific Journal of Mathematic 


\title{
REGULAR SEQUENCES AND LIFTING PROPERTY
}

\author{
M. Herrmann and R. Schmidt
}

Let $A$ be a commutative noetherian ring, $E$ a finite $A$-module and let $M$ be an arbitrary $A$-module. Let $\varphi: E \rightarrow M$ be a homomorphisn of $A$-modules.

In this note we prove in an elementary way that an $M$-sequence $\underline{x}=\left(x_{1}, \cdots, x_{n}\right)$ being taken to lie in the (Jacobson-) radical $\operatorname{rad}(A)$ of $A$, is also an $E$-sequence if $\underline{x} E$ is the contraction $\varphi^{-1}(\underline{x} M)$ of $\underline{x} M$ in $E$.

As a corollary of this lifting property we obtain very easily the so-called delocalization-lemma for regular sequences (also [2], Cor. 1 for local rings $A$ and [4] Chap. I, §4). Then we exemplify that the condition $\varphi^{-1}(x M)=x E$ is not necessary for the statement of our theorem (see Example 3); otherwise it is easily seen that generally the theorem (especially Corollary 2) becomes false without any additional condition (see Examples 1 and 2).

Recall that a sequence $x_{1}, \cdots, x_{n}$ of elements of $A$ is said to be (M-regular or) an $M$-sequence if, for each $0 \leqq i \leqq n-1, a_{i+1}$ is a nonzerodivisor on $M /\left(x_{1}, \cdots, x_{t}\right) M$ and $M \neq\left(x_{1}, \cdots, x_{n}\right) M$.

2. First we consider the case $n=1$.

LEMMA. The notations being as above. Let $x$ be a $M$-regular element in the radical $\operatorname{rad}(A)$ of $A$ and suppose that

$$
\operatorname{ker} \varphi \subseteq x E^{1} \text {. }
$$

Then $x$ is an E-regular element too and $\varphi$ is injective.

Proof. We put $F=\operatorname{ker} \varphi$. Clearly $x$ is $E / F$-regular, hence $x E \cap$ $F=x F$, hence $F=x F$ by (1). Therefore we get $F=0$ by Nakayama's lemma, hence $\varphi$ is injective and $x$ is $E$-regular.

Theorem. Let $E$ be a finite $A$-module, $M$ an arbitrary $A$-module and $\varphi: E \rightarrow M$ a module-homomorphism. Let $\underline{x}=\left(x_{1}, \cdots, x_{n}\right)$ be an $M$-sequence in $\operatorname{rad}(A)$ and suppose that

' We denote by $x E$ or $\underline{x} E$ the product $(x) E$ or $(\underline{x}) E$ respectively, where $(x)$ or $(\underline{x})$ is the ideal generated by $x$ or $x_{1}, \cdots, x_{n}$ respectively. 


$$
\varphi^{-1}(\underline{x} M)=\underline{x} E^{1}
$$

Then $\underline{x}$ is an $E$-sequence and $\varphi$ is injective.

Proof. (By induction on $n$ ): Note that $\operatorname{ker} \varphi \subseteq \varphi^{-1}(\underline{x} M)$, hence the case $n=1$ results from the lemma.

For $n>1$ we put $\underline{x}^{\prime}=\left(x_{1}, \cdots, x_{n-1}\right), E^{\prime}=E / \underline{x}^{\prime} E, M^{\prime}=M / \underline{x}^{\prime} M$ and $\varphi^{\prime}=\varphi \otimes 1_{A / x^{\prime} A}: E^{\prime} \rightarrow M^{\prime}$. Then we have

$$
\operatorname{ker} \varphi^{\prime}=\varphi^{-1}\left(\underline{x}^{\prime} M\right) / \underline{x}^{\prime} E \cap \varphi^{-1}\left(\underline{x}^{\prime} M\right)=\varphi^{-1}\left(\underline{x}^{\prime} M\right) / \underline{x}^{\prime} E \subseteq \varphi^{-1}(\underline{x} M) / \underline{x}^{\prime} E,
$$

hence we get by (2):

$$
\operatorname{ker} \varphi^{\prime} \subseteq \underline{x} E / \underline{x}^{\prime} E=x_{n} \cdot E^{\prime}
$$

Since $x_{n}$ is $M^{\prime}$-regular we are in the situation of the lemma with $x_{n}$ and $\varphi^{\prime}: E^{\prime} \rightarrow M^{\prime}$ instead of $x$ and $\varphi$. Therefore $\varphi^{\prime}$ is injective, i.e.

$$
\varphi^{-1}\left(\underline{x}^{\prime} M\right)=\underline{x}^{\prime} E,
$$

and $x_{n}$ is an $E^{\prime}$-regular element. The sequence $\underline{x}^{\prime}$ is $M$-regular by assumption, hence by (3) and by induction on $n, \underline{x}^{\prime}$ is $E$-regular and $\varphi$ is injective. This concludes the proof.

Corollary 1.1 (Delocalization Lemma, 1. form): Let $A$ be a noetherian ring, $E$ a finite $A$-module and $x_{1}, \cdots, x_{n} \in \operatorname{rad}(A)$. Let $U \subset A$ be the set of nonzerodivisors modulo $\underline{x}$ for $E$. Suppose that $\underline{x}$ is an $E_{U}$-sequence. Then $\underline{x}$ is an E-sequence.

Proof. Let $\varphi: E \rightarrow E_{U}$ be the natural homomorphism. No element of $U$ is zerodivisor for $E / \underline{x} E$, hence $\varphi^{-1}\left(\underline{x} E_{U}\right)=\underline{x} E$, proving the corollary.

It results from the following Corollary 1.2 that the conditions for $\underline{x}$ in the two Corrollaries 1.1 and 1.2 are equivalent.

Corollary 1.2 (Delocalization Lemma, 2. form): Let $E$ be a finitely generated module over $a$ noetherian ring $A$ and $x_{1}, \cdots, x_{r} \in$ $\operatorname{rad}(A)$. If $\underline{x}$ is an $E_{\mathfrak{y}}$-sequence for all $\mathfrak{y} \in A s s(E / \underline{x} E)$, then $\underline{x}$ is an E-sequence.

Proof. Let $M=\bigoplus E_{\mathfrak{y}}$ for $\mathfrak{y} \in A s s(E / \underline{x} E)$, and $\varphi$ the homomorphism $E \rightarrow M$ defined by $u \rightarrow \Sigma_{\eta} \varphi_{\eta}(u)$, where $\varphi_{\eta}$ denotes the natural map $E \rightarrow E_{\mathfrak{n}}$. [Note that Ass $(E / \underline{x} E)$ is a finite set.] 
Since $\underline{x}$ is an $E_{\mathfrak{g}}$-sequence for all $\mathfrak{y} \in \operatorname{Ass}(E / \underline{x} E)$, it must be an $M$-sequence too. We want to apply our theorem to finish the proof. For that we show that $\varphi^{-1}(\underline{x} M)=\underline{x} E$ :

Since $E$ is finitely generated, the submodule $\underline{x} E$ has an irredundant primary decomposition $x E=Q_{1} \cap \cdots \cap Q_{r}$ corresponding to the ideals $\mathfrak{y}_{\mathfrak{t}} \in A s s(E / \underline{x} E)$. Localizing $\underline{x} E$ by any ideal $\mathfrak{y} \in A s s(E / \underline{x} E)$ we obtain [5]:

$$
\varphi_{\mathfrak{y}}^{-1}\left(\left(Q_{\mathfrak{l}}\right)_{\mathfrak{y}}\right)= \begin{cases}Q_{\mathfrak{l}} & \text { if } \mathfrak{y} \mathfrak{l} \subseteq \mathfrak{y} \\ E & \text { if } \mathfrak{y}_{\mathfrak{l}} \not \subset \mathfrak{y},\end{cases}
$$

hence $\cap_{\eta} \varphi_{\eta}^{-1}\left(\underline{x} E_{\mathfrak{\eta}}\right)=x E$.

On the other hand we have $\underline{x} M=\bigoplus_{\mathfrak{n}} \underline{x} E_{\mathfrak{y}}$, hence $\varphi^{-1}(\underline{x} M)=\cap_{\eta} \varphi_{\eta}^{-1}\left(\underline{x} E_{\eta}\right)$. This concludes the proof of the corollary.

3. Now let $f: A \rightarrow B$ be a ring-homomorphism. If $a$ and $b$ are ideals in $A$ and $B$ respectively we define as usual $\mathfrak{a}^{e}$ to be the extension $f(\mathfrak{a}) B$ of $\mathfrak{a}$ and $\mathfrak{b}^{c}$ to be the contraction $f^{-1}(\mathfrak{b})$ of $\mathfrak{b}$.

Corollary 2. Let $f: A \rightarrow B$ be a homomorphism of noetherian rings. Let $a$ be an ideal generated by elements $x_{1}, \cdots, x_{n} \in$ $\operatorname{rad}(A)$. Suppose that $f\left(x_{1}\right), \cdots, f\left(x_{n}\right)$ form a $B$-sequence and suppose that $\mathfrak{a}^{\text {ec }}=\mathfrak{a}$. Then $x_{1}, \cdots, x_{n}$ is an $A$-sequence.

Proof. Regard $B$ as an $A$-module relatively to $f$. We consider the module-homomorphism $\varphi: A \rightarrow B$ given by $\varphi(a)=f(a)$ for all $a \in A$. Then, by assumption all conditions of the theorem are fulfilled, proving the corollary.

Remarks. (i) The proof of Corollary 2 shows that the delocalization Lemma 1.1 or 1.2 respectively can be formulated for rings too.

(ii) If $f: A \rightarrow B$ is faithfully flat then any sequence $x_{1}, \cdots, x_{n} \in$ $\operatorname{rad}(A)$ is $A$-regular $\Leftrightarrow f\left(x_{1}\right), \cdots, f\left(x_{n}\right)$ is $B$-regular. This well-known statement (s. [4] or [3]) is an easy consequence of Corollary 2: $f$ is faithfully flat says that $f$ is flat and the induced map ${ }^{a} f: \operatorname{Spec} B \rightarrow \operatorname{Spec} A$ is surjective. But if $f$ is flat then the last condition is equivalent to $\mathfrak{a}^{e c}=\mathfrak{a}\left(\mathrm{s}\right.$. [1], p. 45), where $a$ is generated by $x_{1}, \cdots, x_{n}$. Hence Corollary 2 works for $\Leftarrow$; the other direction is trivial.

(iii) Let $B$ be a surjectively-free $A$-algebra [i.e. $A=\Sigma_{\psi} \psi(B)$, where $\psi$ runs over $\left.\operatorname{Hom}_{A}(B, A)\right]$. Then for any ideal $\mathfrak{a}$ of $A$ one has

$$
\mathfrak{a}^{e c}=\mathfrak{a} B \cap A=\mathfrak{a},
$$

and the induced map $\operatorname{Spec} B \rightarrow \operatorname{Spec} A$ is surjective; see [5], (5, E), p. 37 . 
4. We are indebted to L. Badescu for pointing to the following

Example 1. Consider the ring

$$
R=\{f \in k[x, y] \mid f(1,0)=f(-1,0)\} \subset k[x, y],
$$

where $k$ denotes say the field of complex numbers. Then $R$ is the finitely generated subring $k\left[x-x^{3}, x^{2}, x y, y\right]$ of $k[x, y]$; clearly $k[x, y]$ is integrally dependent on $R$ and with the same quotient field. Therefore $Y:=\operatorname{Spec} R$ is not normal. Write $X$ for the normal affine variety $\operatorname{Spec}(k[x, y]) \cong k^{2}$. Let the inclusion of $R$ in $k[x, y]$ define the proper morphism

$$
\pi: X \rightarrow Y
$$

Then if $x_{1}, x_{2}$ are the points $(1,0)$ and $(-1,0) \in k^{2}$, we have $\pi\left(x_{1}\right)=$ $\pi\left(x_{2}\right)=: y_{0}$, and

$$
\text { res } \pi: X-\pi^{-1}\left\{y_{0}\right\} \rightarrow Y-\left\{y_{0}\right\}
$$

is an isomorphism. In particular, $Y$ is normal at all points except $y_{0}$ [this is also clear by the connectedness theorem, because $\pi^{-1}\left\{y_{0}\right\}$ is not connected]. To be more in detail, take

$$
v_{1}=1-x^{2}, v_{2}=x y, v_{3}=y, v_{4}=x-x^{3}
$$

Then

$R=$

$k\left[v_{1}, v_{2}, v_{3}, v_{4}\right] /\left(v_{4} v_{3}-v_{2} v_{1} ; v_{2}^{2}-v_{3}^{2}+v_{1} v_{3}^{2} ; v_{4}^{2}+v_{1}^{3}-v_{1}^{2} ; v_{1} v_{2}-v_{2} v_{4}-v_{1}^{2} v_{3}\right)$.

So $Y$ can be regarded as an affine surface in $k^{4}$, which is nonsingular in codimension 1, but not normal in the origin (corresponds to the point $y_{0} \in \operatorname{Spec} R=Y$ ). Therefore $0_{Y, y_{0}}$ is not a Cohen Macaulay-ring by the criterion of normality, [3], 5.8.6.

We fix the notations:

$$
\begin{array}{lll}
A=0_{Y, y_{0}}=R_{\mathfrak{y}} & \text { with } & \mathfrak{y}=(x-1, y) \cap R=(x+1, y) \cap R \\
B=0_{X, x_{1}}=k[x, y]_{\mathfrak{y}} & \text { with } \quad \mathfrak{y}=(x-1 ; y)
\end{array}
$$

$f: A \rightarrow B$ the corresponding local homomorphism; $a_{1}=$ $(x-1)(x+1), a_{2}=y$ in $A$ and $f\left(a_{1}\right)=(x-1)(x+1), f\left(a_{2}\right)=y$ regarded as being in $B$. Then $B$ is a regular local ring, and 
$f\left(a_{1}\right), f\left(a_{2}\right)$ generate its maximal ideal $m_{B}$. Since depth $A \lessgtr \operatorname{dim} A=2$ the sequence $a_{1}, a_{2}$ will not be $A$-regular. And indeed we have $\mathfrak{a}^{e}=m_{B}$ and $a^{e c} m_{A} \neq a$.

EXAMPLE 2. Let $(A, m)$ be a one-dimensional local noetherian ring which is not a Cohen Macaulay-ring. Let $x \in \mathrm{m}$ be a parameter of $A$ and $\mathfrak{y}$ a minimal prime over-ideal of zero in $A$. Take $B=A / \mathfrak{y}$. Then by assumptions $f(x)$ is $B$-regular, but $x$ is not $A$-regular. And we have $\mathfrak{a}^{\text {ec }} \neq \mathfrak{a}$ (otherwise $\mathfrak{y}$ would be zero).

EXAMPLE 3. Let $(A, \mathfrak{m})$ be a local Cohen Macaulay-ring of dimension 1 which is not regular. Then the maximal ideal $m$ can be generated in this way:

$$
\mathrm{m}=x A+m_{1} A+\cdots+m_{r} A
$$

where $\boldsymbol{x}$ denotes an $\boldsymbol{A}$-regular element.

Let $a$ be the ideal generated by $x$ and $B=A\left[m_{1} / x\right] \subset A_{x}$. Since $x$ is $A$-regular the natural homomorphism $f: A \rightarrow B$ is injective, and clearly $\boldsymbol{x}$ is $\boldsymbol{B}$-regular.

But now we have $\mathfrak{a}^{e}=x B=\mathfrak{m} B$, hence $\mathfrak{a}^{e c}=\mathfrak{m} B \cap A=\mathfrak{m}$, hence $\mathfrak{a}^{e c} \neq \mathfrak{a}$ because $A$ is not regular.

This example shows that condition (2) is not necessary for the statement of the theorem.

\section{REFERENCES}

1. M. F. Atiyah and I. G. MacDonald, Introduction to Commutative algebra, Addison-Wesley, London , 1969.

2. D. Eisenbud, M. Herrmann and W. Vogel, Remarks on regular sequences, Nagoya Math. J., 67 (1977), 177-180.

3. A. Grothendieck, Eléments de géometrie algébrique, I.H.E.S. Publ. Math. Nr. 24, Paris, 1965.

4. M. Herrmann, R. Schmidt and W. Vogel, Normale Flacheit, charakteristische Funktionen und Cohen Macaulay-Strukturen-Teubner Texte Leipzig 1977.

5. H. Matsumura, Commutative Algebra, W. A. Benjamin, Inc., New York, 1970.

Received April 20, 1977.

HUMBOLDT UNIVERSITY

DDR-108 BERLIN 



\section{PACIFIC JOURNAL OF MATHEMATICS}

\section{EDITORS}

RICHARD ARENS (Managing Editor)

University of California

Los Angeles, CA 90024

R. A. BeAumont

University of Washington

Seattle, WA 98105

C. C. MOORE

University of California

Berkeley, CA 94720
J. DUGUNDJI

Department of Mathematics

University of Southern California

Los Angeles, CA 90007

R. FINN AND J. MILGRAM

Stanford University

Stanford, CA 94305

\section{ASSOCIATE EDITORS}
E. F. BECKENBACH
B. H. NEUMANN
F. WOLF
K. YoshidA

\section{SUPPORTING INSTITUTIONS}

UNIVERSITY OF BRITISH COLUMBIA

UNIVERSITY OF SOUTHERN CALIFORNIA

CALIFORNIA INSTITUTE OF TECHNOLOGY

STANFORD UNIVERSITY

UNIVERSITY OF CALIFORNIA

UNIVERSITY OF HAWAII

MONTANA STATE UNIVERSITY

UNIVERSITY OF TOKYO

UNIVERSITY OF NEVADA

UNIVERSITY OF UTAH

NEW MEXICO STATE UNIVERSITY

OREGON STATE UNIVERSITY

UNIVERSITY OF OREGON

OSAKA UNIVERSITY

WASHINGTON STATE UNIVERSITY

UNIVERSITY OF WASHINGTON

AMERICAN MATHEMATICAL SOCIETY

The Supporting Institutions listed above contribute to the cost of publication of this Journal, but they are not owners or publishers and have no responsibility for its contents or policies.

Mathematical papers intended for publication in the Pacific Journal of Mathematics should be in typed form or offset-reproduced (not dittoed), double spaced with large margins. Underline Greek letters in red, German in green, and script in blue. The first paragraph or two must be capable of being used separately as a synopsis of the entire paper. Items of the bibliography should not be cited there unless absolutely necessary, in which case they must be identified by author and Journal, rather than by item number. Manuscripts, in duplicate, may be sent to any one of the four editors. Please classify according to the scheme of Math. Reviews, Index to Vol. 39. All other communications should be addressed to the managing editor, or Elaine Barth, University of California, Los Angeles, California, 90024.

100 reprints are provided free for each article, only if page charges have been substantially paid. Additional copies may be obtained at cost in multiples of 50 .

The Pacific Journal of Mathematics is issued monthly as of January 1966. Regular subscription rate: $\$ 72.00$ a year (6 Vols., 12 issues). Special rate: $\$ 36.00$ a year to individual members of supporting institutions.

Subscriptions, orders for numbers issued in the last three calendar years, and changes of address should be sent to Pacific Journal of Mathematics, 103 Highland Boulevard, Berkeley, California, 94708.

PUBLISHED BY PACIFIC JOURNAL OF MATHEMATICS, A NON-PROFIT CORPORATION

Printed at Jerusalem Academic Press, POB 2390, Jerusalem, Israel. 


\section{Pacific Journal of Mathematics}

\section{Vol. 75, No. $2 \quad$ October, 1978}

Susan Jane Zimmerman Andima and W. J. Thron, Order-induced

topological properties ................................... 297

Gregory Wade Bell, Cohomology of degree 1 and 2 of the Suzuki groups . . 319

Richard Body and Roy Rene Douglas, Rational homotopy and unique

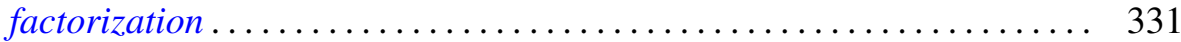

Frank Lewis Capobianco, Fixed sets of involutions ................. 339

L. Carlitz, Some theorems on generalized Dedekind-Rademacher sums .... 347

Mary Rodriguez Embry and Alan Leslie Lambert, The structure of a special class of weighted translation semigroups .....................

Steve Ferry, Strongly regular mappings with compact ANR fibers are

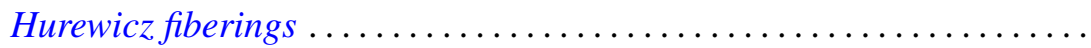

Ivan Filippenko and Marvin David Marcus, On the unitary invariance of the

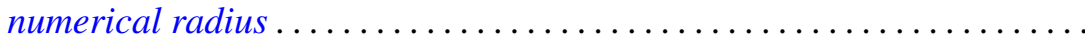

$\mathrm{H}$. Groemer, On the extension of additive functionals on classes of convex

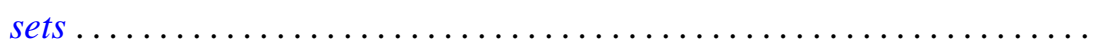

Rita Hall, On the cohomology of Kuga's fiber variety ............... 411

H. B. Hamilton, Congruences on $\mathrm{N}$-semigroups ................. 423

Manfred Herrmann and Rolf Schmidt, Regular sequences and lifting

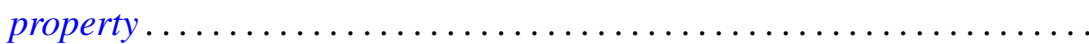

James Edgar Keesling, Decompositions of the Stone-Čech compactification which are shape equivalences .....................

Michael Jay Klass and Lawrence Edward Myers, On stopping rules and the expected supremum of $S_{n} / T_{n}$

Ronald Charles Linton, $\lambda$-large subgroups of $C_{\lambda}$-groups

William Owen Murray, IV and L. Bruce Treybig, Triangulations with the free cell property ............................

Louis Jackson Ratliff, Jr., Polynomial rings and $H_{i}$-local rings ...

Michael Rich, On alternate rings and their attached Jordan rings....

Gary Sampson and H. Tuy, Fourier transforms and their Lipschitz

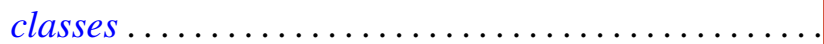

Helga Schirmer, Effluent and noneffluent fixed points on dendrites ...

Daniel Byron Shapiro, Intersections of the space of skew-symmetric maps

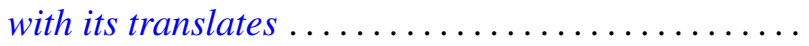

Edwin Spanier, Tautness for Alexander-Spanier cohomology ...

Alan Stein and Ivan Ernest Stux, A mean value theorem for binary digits ...

Franklin D. Tall, Normal subspaces of the density topology . .

William Yslas Vélez, Prime ideal decomposition in $F\left(\mu^{1 / p}\right) \ldots$ 